\title{
Análisis Espectroscópico en la Pigmentación de Dientes para Prótesis por Contacto con Café
}

\author{
Laura Susana Acosta-Torres ${ }^{1}$, Karen Castaño-González ${ }^{2}$, Carmen Vázquez-Ramos ${ }^{3}, V^{\prime}$ 'tor M. Castaño ${ }^{1 a}$ \\ y Genoveva Hernandez-Padrón ${ }^{3}$
}

${ }^{I}$ Departamento de Ingeniería Molecular de Materiales, ${ }^{2}$ Licenciatura en Tecnología, ${ }^{3}$ Departamento de Nanotecnología, Centro de Física Aplicada y Tecnología Avanzada, Universidad Nacional Autónoma de México, Campus Juriquilla, Boulevard Juriquilla No. 3001, Juriquilla, Querétaro, México, C.P. 76230.

${ }^{a}$ Cumpliendo una estancia sabática en la Universidad Autónoma de Querétaro.

Recibido 27 de Octubre de 2011, Aceptado 15 de Noviembre de 2011

\section{Resumen}

Hoy en día el consumo de café ente la población mexicana, es una práctica constante. Los consumidores no solo adquieren café de origen nacional sino también de importación. El café ha resultados tener grandes propiedades benéficas para el organismo, sin embargo una desventaja es la pigmentación que produce en los dientes. En México el $60 \%$ de los adultos mayores de 60 años son portadores de prótesis removibles, las cuales son confeccionadas con dientes artificiales elaborados con diferentes tipos de resinas. Los materiales de las bases de las prótesis, así como los dientes de resina sufren mayor pigmentación al estar en contacto con los fluidos orales, por lo que en el presente estudio se evaluó la pigmentación de dientes de resina después de someterlos a 30 y 60 ciclos en contacto con café de Guatemala y Veracruz. Los resultados mostraron que el café de Guatemala produce mayor pigmentación y pérdida de brillo en los dientes de resina. Ambos tipos de café no producen cambio significativo en las propiedades mecánicas de los dientes para prótesis.

\section{Introducción}

México es hoy en día un productor e importador de café; aun cuando el café no es uno de los productos principales de la dieta, es un producto marcado por el hábito de consumo a nivel mundial [1].

Es conocido que el esmalte de los dientes puede ser afectado gradualmente por algunos alimentos y bebidas cotidianas, básicamente sustancias ácidas, tabaco, y no podríamos dejar a la cafeína, por mencionar algunos [2]. El deterioro de las estructuras dentales lleva a muchos pacientes a la utilización de prótesis, [3] con las que estéticamente se cubren las expectativas del paciente, sin embargo el consumo de bebidas ácidas que contienen cafeína, puede tener graves consecuencias en el cambio de color de los dientes protésicos [4].

El café contiene compuestos químicos como los alcaloides entre los que destaca la cafeína; sustancia psicoactiva que estimula la transmisión de los impulsos entre las células nerviosas [5], además de ser un compuesto capaz de pigmentar los dientes.

Cafeína es el nombre común para la trimetilxantina (1,3,7-trimetilxantina). Ésta es producida naturalmente por varias plantas, entre ellas el café, la guaraná, la yerba mate, el cacao y el té. Para las plantas la cafeína actúa como un pesticida natural, paralizando y matando insectos que intentan alimentarse de la planta [6]. La cafeína también es consumida en bebidas de cola, chocolate y té [7].

Se considera que $100 \mathrm{mg}$ es una dosis normal de cafeína y es aproximadamente la cantidad encontrada en una taza de café. Sin embargo algunas personas consumen más de 300 mg de café todos los días, lo que convierte al café en una de los pigmentos naturales más comunes de los dientes.

El objetivo de esta investigación fue evaluar el deterioro mediante espectroscopia Raman e infrarroja de los dientes por el contacto de dos tipos de café con la pigmentación y el comportamiento mecánico en dientes de resina compuesta utilizados en la elaboración de prótesis removibles.

\section{Parte Experimental}

En este trabajo se realizó la simulación de ingesta de dos tazas de café diarias durante 30 días, para lo cual se utilizaron 18 dientes de resina compuesta marca VivodentPE (Ivoclar, Vivadent, Liechtenstein), café en polvo de Guatemala y Veracruz.

Los dientes se dividieron en 3 grupos, un grupo por cada tipo de café y un grupo control (sin estar en contacto con café).

Se prepararon $13 \mathrm{~g}$ de cada café en $200 \mathrm{~mL}$ de agua y calentaron a $55^{\circ} \mathrm{C}$. El pH de cada solución de café fue medido usando un pH-metro ORION, E.U. Las mediciones de $\mathrm{pH}$ se realizaron por triplicado. Los dientes se mantuvieron sumergidos de forma individual en $2 \mathrm{~mL}$ de café durante ciclos de 1 min por 7 repeticiones (sorbos promedio por taza de café). En total se realizaron 60 ciclos por cada grupo de café.

Las muestras de cada tipo de café se analizaron mediante espectroscopía de infrarrojo y Raman dispersivo, y fueron comparados con la cafeína pura (Sigma-Aldrich).

$\mathrm{El}$ análisis de espectroscopia infrarroja fue realizado en un espectrofotómetro FT-IR Bruker Vector 33 en una longitud de láser de $1064 \mathrm{~nm}$, en el intervalo de número de onda de 4000 a $400 \mathrm{~cm}^{-1}$. Las muestras fueron corridas mediante la técnica de transmitancia, en las cuales se prepararon pastillas delgadas y transparentes de una mezcla de muestra con $\mathrm{KBr}$, y medidas a 32 barridos.

Para identificar el cambio de la estructura en la resina debida a la pigmentación, los dientes se caracterizaron mediante un espectrofotómetro Raman dispersivo Bruker modelo Senterra acoplado con un microscopio óptico, en un equipo Senterra marca Bruker, con un láser de longitud de onda de $785 \mathrm{~nm}$; se utilizaron $10 \mathrm{~s}$ de tiempo de integración, con una potencia del láser de $100 \mathrm{~mW}$, en un intervalo de número de onda de 80 a $3500 \mathrm{~cm}^{-1}$. Los análisis espectroscópicos se realizaron cuando los dientes estuvieron en contacto con café durante 30 y 60 ciclos capturando imágenes de las zonas pigmentadas. 
El cambio de color de los dientes se evaluó antes y después de las inmersiones en café; utilizando un colorímetro o guía de color (Ivoclar, Vivadent). Los dientes se colocaron sobre un lienzo azul y bajo luz natural se compararon con la guía de color para identificar el color al inicio del estudio; el cambio de color se fue monitoreando con el transcurso de los ciclos en contacto con el café hasta observa un cambio notable.

La brillantez y pérdida de esmalte se analizaron mediante espectrometría UV-Vis con un equipo Spectronic* Genesys" 2PC.

Posteriormente, los dientes se sometieron al ensayo de profundidad de penetración para evaluar dureza. Se utilizó una punta aguda para penetrar los dientes, ajustada a una máquina universal de pruebas mecánicas (Adamel Lhomargy DY.22; Francia) con una velocidad de penetración de $1 \mathrm{~mm} / \mathrm{min}$, aplicando una carga de máximo 200 N. Se realizaron dos indentaciones en diferentes zonas de cada diente, evaluando 4 dientes por grupo.

Posteriormente, los dientes ensayados fueron preparados desgastando la superficie superior e inferior con papel abrasivo, hasta obtener discos de $4 \mathrm{~mm}$ de espesor, los cuales se sometieron a 60 ciclos de inmersión en contacto con café de Veracruz y Guatemala y se evaluaron en la máquina universal de pruebas mecánicas para hacer la prueba de resistencia a la compresión. El experimento se realizó a una velocidad de $5 \mathrm{~mm} / \mathrm{min}$; como grupo control se analizaron los dientes sin estar en contacto con café.

El análisis estadístico de los resultados obtenidos se realizó medianteANOVA de Una Vía $(p<0.05)$.

\section{Resultados y discusión}

En la figura 1 se observa los espectra de infarrojo, en donde se compara la cafeína pura (Sigma Aldrich) y los dos grupos de café en estudio: Veracruz y Guatemala. Los resultados muestran presencia de "huellas digitales" muy semejantes entre los tres espectra; mostrando la banda de absorción de la cafeína en el intervalo de 1740 a $1750 \mathrm{~cm}^{-1}$ dependiendo del café analizado (Figura 2).

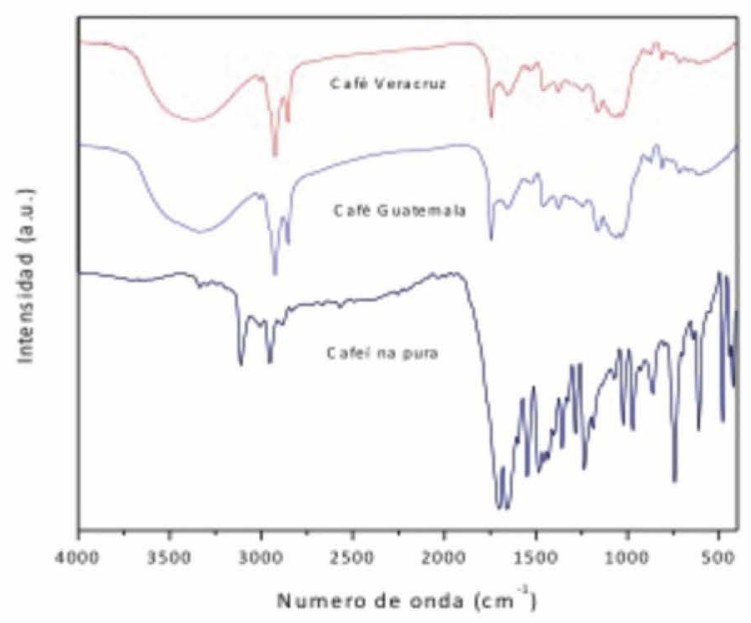

Figura 1. Espectro FT-IR de café de Veracruz, café de Guatemala y cafeína pura.

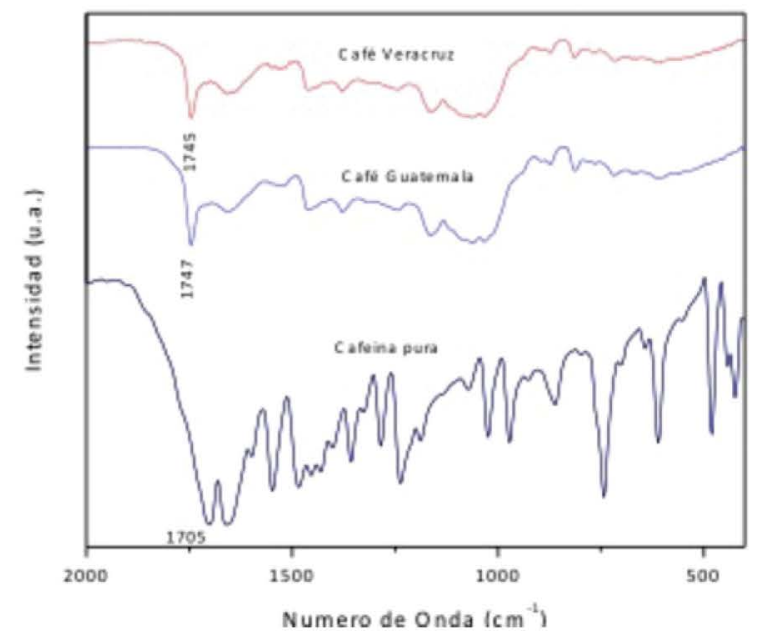

Figura 2. Espectra FTIR en el intervalo de absorción de 2000$400 \mathrm{~cm}^{-1}$.

Los resultados de espectroscopía Raman dispersivo para 30 ciclos en contacto con café no mostraron cambios significativos en la estructura química de los dientes de resina (Figura $3 a$ y $3 b$ ).

Dentro de los resultados espectroscópicos después de 60 ciclos de los dientes en contacto con el café de Guatemala, se observa una banda de absorción en $1655 \mathrm{~cm}^{-1}$, característica de la cafeína (Figura 3b); mientras que los espectros de los dientes en contacto con café de Veracruz muestran la misma banda pero en menor intensidad.
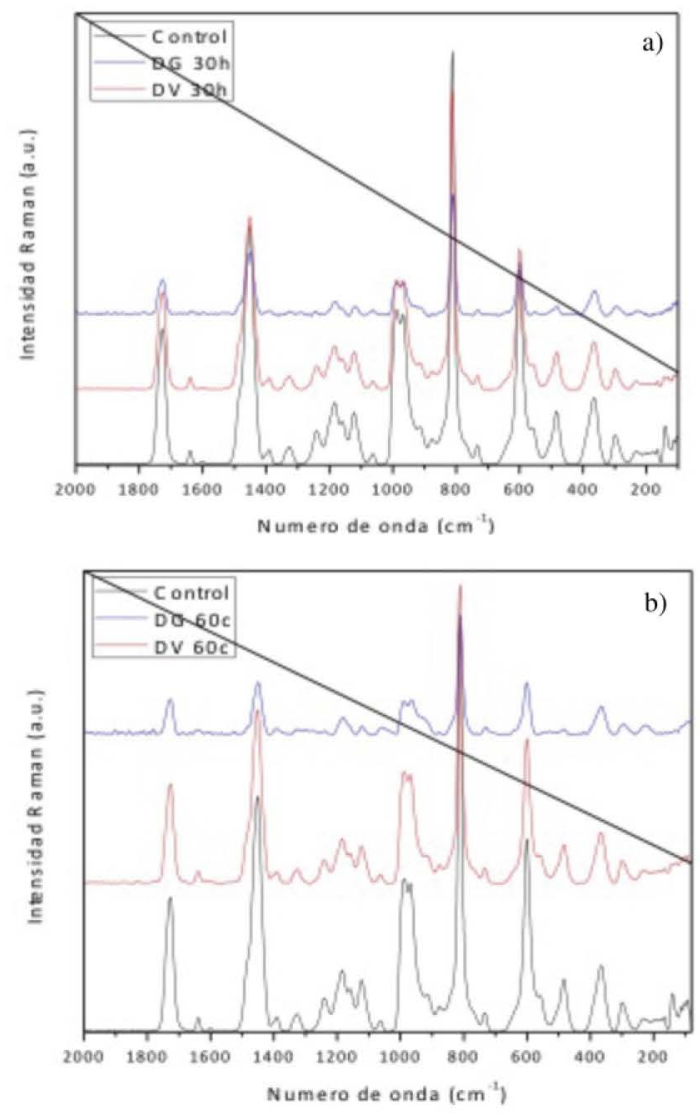

Figura 3. Espectro Raman dispersivo de los dientes en contacto con café de Guatemala (DG) y Veracruz (DV) después de a) 30 ciclos y b) 60 ciclos. 


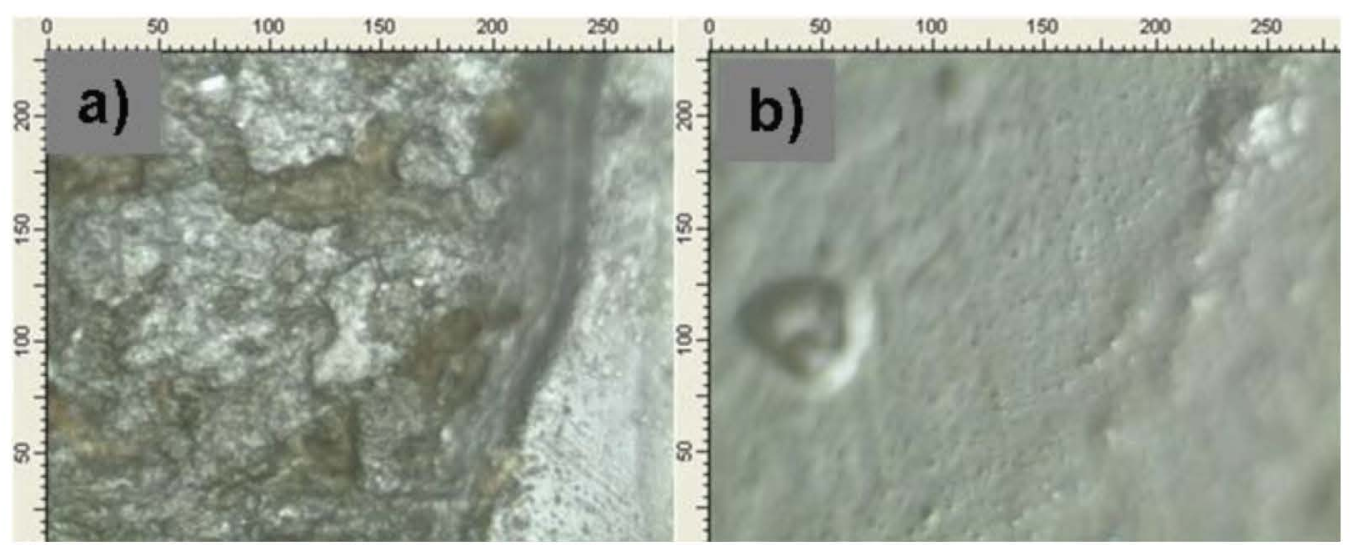

Figura 4. Microfotografía (20x) de los dientes de resina después de haber sido sometidos a 60 ciclos en contacto con café (a) de Guatemala y b) de Veracruz.

Las microfotografías analizadas obtenidas por Raman comprueban un deterioro en la morfología superficial de los dientes a 60 ciclos, los cual coincide con la presencia de cafeína debida al contacto con café de Guatemala, además de que muestra mayor cantidad de zonas pigmentadas (Figura 4a), a diferencia de los dientes con café de Veracruz (Figura 4b) que prácticamente no presenta cambios superficiales.

La presencia de zonas pigmentadas de los dientes puede ser debido a que el $\mathrm{pH}$ de ambos tipos de café muestra un grado de acidez menor al presente en la cavidad oral ( $\mathrm{pH} 7$ 7.4) [8]; lo cual puede degradar la estructura de los dientes y permitir la penetración de moléculas de café al interior de los dientes [2]. Los valores de $\mathrm{pH}$ para las soluciones de café fueron para el café de Guatemala de 5.15 y para el café de Veracruz de 5.05.

Se realizaron análisis de absorción de UV-Vis, mostrando que los dientes a 60 ciclos para el café de Guatemala, presentan una mayor pérdida de brillantez, en comparación con el grupo de dientes expuesto al café de Veracruz y al grupo control (Figura 5).

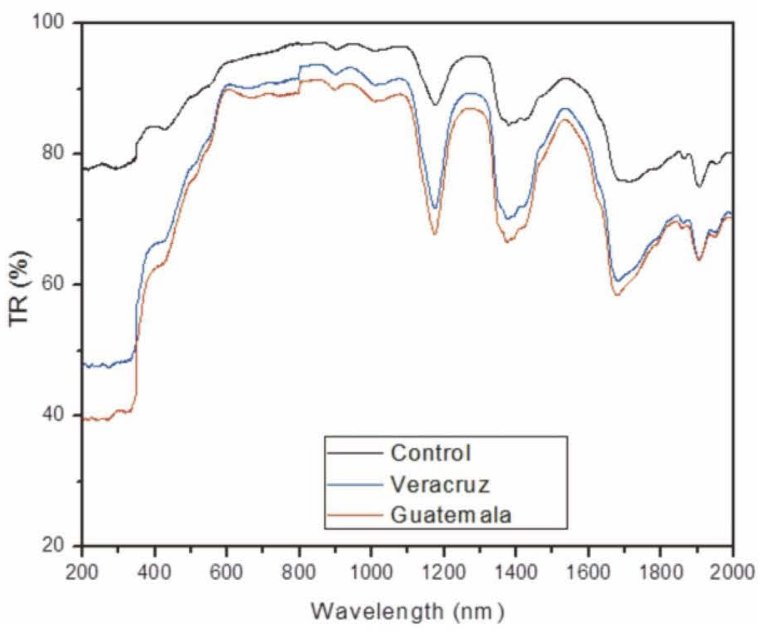

Figura 5. Espectro UV-Vis de los dientes de resina después de 60 ciclos en contacto con café de Guatemala y Veracruz.
En la figura 6 se observa el cambio de color de los dientes en contacto con café de Guatemala (Figura 6a) al comparar con el diente seleccionado del colorímetro $(1 \mathrm{~A} / 120)$. La comparación del diente del colorímetro con el diente sometido a contacto con café de Veracruz no mostró cambio significativo de color (Figura 6b).
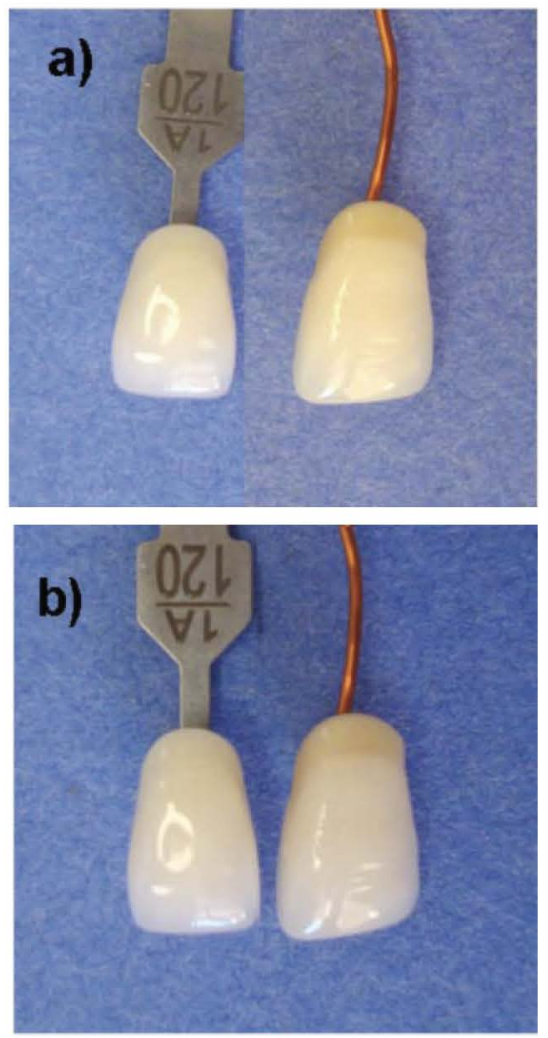

Figura 6. Fotografías comparativas del colorímetro $(1 \mathrm{~A} / 120)$ con los dientes evaluados después de 60 ciclos en contacto con a) café de Guatemala, los cuales muestran cambio de color y b) café de Veracruz, no mostrando diferencia significativa. 
La figura 7 se muestran los resultados de la prueba de profundidad de penetración, los cuales no mostraron diferencia estadísticamente significativa $(\mathrm{p}>0.05)$, indicando que el consumo de estos tipos de café no afecta la dureza de los dientes después de estar en contacto durante 60 ciclos.

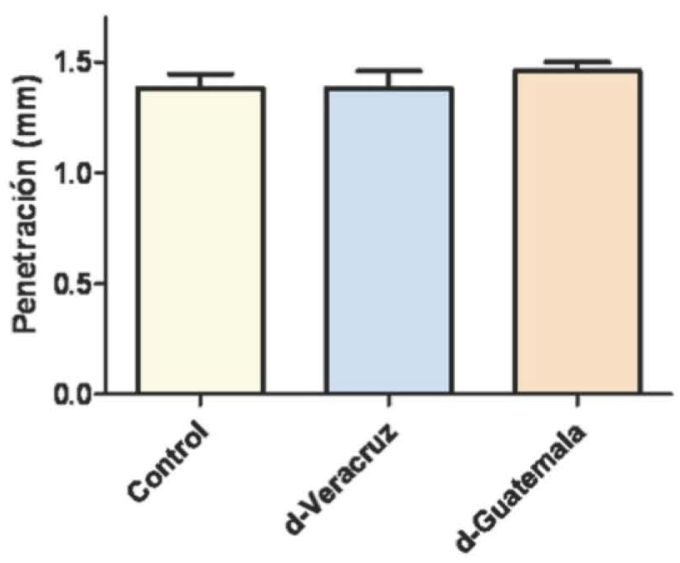

Figura 7. Valores de penetración de los dientes analizados después de someterlos a 60 ciclos en contacto con café $n=8,(p>$ $0.05)$. El grupo control fueron dientes sin contacto con café.

Al realizar pruebas de compresión, los dientes no se rompieron completamente, la máquina los comprimió pero éstos sólo llegaron a fisurarse un poco en los bordes y el espesor de cada diente se vió reducido por uno o dos milímetros. En la Figura 8 se observa el cambio dimensional obtenido con la diferencia de altura inicial respecto a la final de cada diente. Se aplicó ANOVA de Una Vía y Tukey Test y el análisis indica que el grupo de dientes en contacto con café de Guatemala fue el que sufrió mayor cambio dimensional al ser comparado con el grupo de dientes en contacto con café de Veracruz $(\mathrm{p}<0.05)$. La carga máxima aplicada para todas las muestras fue de $2,500 \mathrm{~N}$.

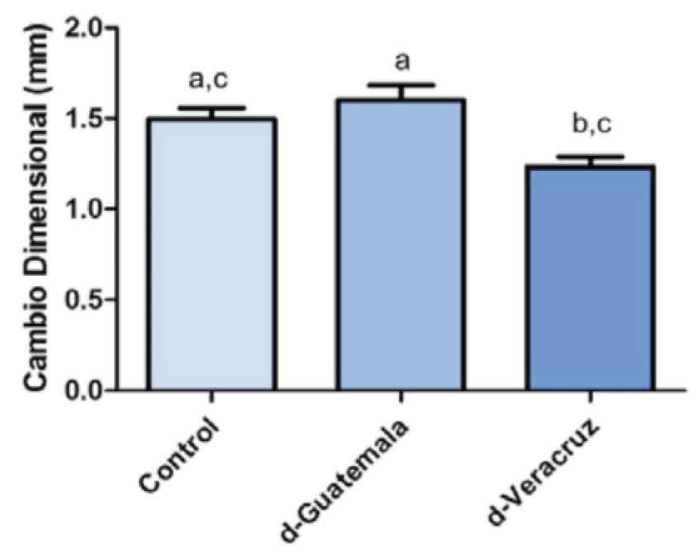

Figura 8. Cambio dimensional de dientes de acrílico (60 ciclos de inmersión con café) al aplicar una Fuerza compresiva máxima de 2,500 N. $\mathrm{n}=6$; ANOVA de Una Vía $\mathrm{p}<0.05$.

\section{Conclusiones}

A partir de los resultados espectroscópicos tomando como referencia el espectro obtenido de la base de datos [9] podemos concluir que la cafeína analizada de ambas muestras de café comparada con la cafeína pura muestra pequeños corrimientos en la banda de absorción característica de la cafeína debido a factores como la humedad, $\mathrm{pH}$ y temperatura en las que se hicieron las mediciones, así como la región de procedencia del café.

El café de Guatemala muestra pigmentación de la estructura dental a nivel microscópico y al ser comparado mediante colorimetría. Esto puede deberse a que el $\mathrm{pH}$ del café de Guatemala y del café de Veracruz muestra acidez significativa, lo cual puede producir deficiencias en la estructura de los dientes para prótesis.

En tanto el efecto del contacto del café no mostró diferencias significativas en la prueba de penetración al estar en contacto con el café de Guatemala ni con el café de Veracruz.

Por otra parte se observó que mantener los dientes de resina en contacto con café de Guatemala disminuyó tanto su resistencia compresiva como la brillantez en el esmalte, mientras que los dientes en contacto con café de Veracruz no sufrieron modificaciones, lo cual puede atribuirse a la acidez de café.

Se requieren de realizar estudios con diferentes muestras de café de varias regiones territoriales, para confirmar que la concentración de cafeína sumada al $\mathrm{pH}$ de diferentes tipos de café son los posibles responsables de la pigmentación y deterioro microscópico de los dientes para prótesis dentales, ya que el $\mathrm{pH}$ es un factor importante en la calidad del buen café gourmet.

\section{Agradecimientos}

Al Ing. Gerardo Fonseca Hernández, M. en C. Carmen Peza Ledesma y Dra. Marina Vega por su excelente apoyo técnico.

\section{Bibliografía}

1. Kang J., Gu H., Zhong L., Hu Y, Liu F. (2011) Spectrochum. Acta Part A. 78: 757-762.

2. M Daglia. (2007) JAgricol Food Chem. 55:10208-10213.

3. H. Corrine. (1996) JProsthet Dent. 75:14-17.

4. M. Daglia. (2002) JAgricol Food Chem. 50:1225-1229.

5. Singh R. B., Wechter A. M., Hu Y., Lafontaine C. (1998) Biochem Edu. 26:243-247.

6. A. M. Helmenstine. "Caffeine chemistry. What is caffeine and how does it work", 2012.

http://chemistry.about.com/od/moleculescompounds/a/ caffeine.htm

7. J.P. Bezerra. (2008) JPeriodontol. 79:2356-2360.

8. Spectral Database for Organic Compounds SDBS http://riodb01.ibase.aist.go.jp/sdbs/cgi-bin/cre_index.cgi

9. M. Lee, J. D. Lambert, S. Prabhu. (2004) Cancer Epidemiology, Biomarkers \& Prevention. 13:1132-137. 